\title{
Géolinguistique
}

$21 \mid 2021$

Varia

\section{L'APV - Atlas des patois valdôtains e il suo primo volume (APV/1: Le lait et les activités laitières): un progetto scientifico, un prodotto editoriale}

L'APV - Atlas des patois valdôtains et son premier volume (APV/1 : Le lait et les activités laitières) : un projet scientifique, un produit éditorial APV - Atlas des patois valdôtains and Its First Volume (APV/1: Milk and Dairy Activities: A Scientific Project, an Editorial Product

\section{Gianmario Raimondi}

\section{OpenEdition}

\section{Journals}

Edizione digitale

URL: https://journals.openedition.org/geolinguistique/5967

DOI: 10.4000/geolinguistique.5967

ISSN: 2650-8176

\section{Editore}

UGA Éditions/Université Grenoble Alpes

\section{Edizione cartacea}

ISBN: 978-2-37747-303-8

ISSN: 0761-9081

\section{Notizia bibliografica digitale}

Gianmario Raimondi, «L'APV - Atlas des patois valdôtains e il suo primo volume (APV/1: Le lait et les activités laitières): un progetto scientifico, un prodotto editoriale», Géolinguistique [Online], 21 | 2021, online dal 06 décembre 2021, consultato il 19 décembre 2021. URL: http://journals.openedition.org/ geolinguistique/5967 ; DOI: https://doi.org/10.4000/geolinguistique.5967

Questo documento è stato generato automaticamente il 19 décembre 2021.

Géolinguistique 


\section{L'APV - Atlas des patois valdôtains e il suo primo volume (APV/1: Le lait et les activités laitières): un progetto scientifico, un prodotto editoriale}

L'APV - Atlas des patois valdôtains et son premier volume (APV/1: Le lait et les activités laitières) : un projet scientifique, un produit éditorial APV - Atlas des patois valdôtains and Its First Volume (APV/1: Milk and Dairy Activities: A Scientific Project, an Editorial Product

\section{Gianmario Raimondi}

\section{Introduzione}

1 L'APV - Atlas des patois valdôtains ha pubblicato finalmente nel 2020 il suo primo volume $(\mathrm{APV}, 2020)^{1}$, grazie al lavoro congiunto dell'équipe del BREL-Bureau Régional pour l'Ethnologie et la Linguistique (struttura di ricerca che fa capo all'Assessorato ai Beni Culturali della Regione Autonoma Valle d'Aosta) e dei ricercatori dell'Università della Valle d'Aosta, riannodando in questo modo i fili di un lavoro cominciato quasi cinquant'anni fa.

2 Il volume è stato presentato alla comunità scientifica e alla comunità locale in occasione di una giornata di studi che si è tenuta online il 6 maggio $2021^{2}$. Nel contributo, dopo una breve contestualizzazione storica ed epistemologica di questo progetto di lungo corso, coglieremo l'occasione per soffermarci su alcuni aspetti di metodo che risultano complementari a quanto già proposto in questa stessa sede editoriale (Raimondi, 2019b) a proposito della struttura dell'opera, in particolare rispetto agli aspetti della copertura geolinguistica dell'atlante e di alcune originalità che si riferiscono all'APV come "progetto scientifico» e come, al tempo stesso, «prodotto editoriale». 


\section{La storia del progetto APV}

Abbiamo già rilevato altrove (Raimondi, 2019b: 1-3) che i semi iniziali del progetto APV germinano nel medesimo clima culturale da cui in Francia scaturisce anche l'idea degli atlas par région, ovvero nel contesto che, all'interno della geolinguistica europea (soprattutto francese, svizzera e italiana), determina il passaggio dagli atlanti nazionali e generalisti (ALF, AIS, ALI) a quelli cosiddetti «di seconda generazione», in cui le realtà regionali diventano l'oggetto elettivo di un'indagine dialettologica maggiormente dettagliata, rispetto alla micro-variazione diatopica, al rapporto tassonomico fra lingua e cultura materiale in senso etno-antropologico (le «parole» e le «cose» della diade jaberg-judiana), al rapporto fra dato dialettologico e contesto sociolinguistico dei punti e delle aree indagate.

4 Aggiungeremo qui che è proprio in Valle d'Aosta che l'idea di un Atlante linguistico del francoprovenzale cisalpino viene proposta la prima volta, alle Journées d'études francoprovençales organizzate nel 1972 ad Aosta dal neonato Centre d'études francoprovençal. Nel Centre, per impulso del suo promotore (lo scrittore ed etnologo valdostano René Willien, cui poi il centro verrà intitolato) si incontravano allora le scuole dialettologiche di Grenoble (Gaston Tuaillon), di Neuchâtel (Ernest Schule) e di Torino (Corrado Grassi e Tullio Telmon) ed è nel segno del loro lavoro che vanno ricercate le radici scientifiche e anche l'impostazione metodologica del progetto.

Di lì a poco, il progetto iniziale (che, come il nome suggerisce, prevedeva l'indagine congiunta delle parlate francoprovenzali piemontesi e valdostane) si sdoppia per ragioni gestionali collegate alle differenti politiche di promozione culturale delle due regioni italiane. In Piemonte, esso diventerà l'ALEPO - Atlante Linguistico ed Etnografico del Piemonte Occidentale, diretto da Tullio Telmon e Sabina Canobbio all'Università di Torino, che includerà quindi anche l'area dell'occitano cisalpino. In Valle d'Aosta il progetto verrà invece preso in carica direttamente dall'amministrazione regionale, anche attraverso la successiva istituzione del BREL - Bureau régional pour l'ethnologie et la linguistique negli anni Ottanta, e darà vita appunto all'APV - Atlas des patois valdotains.

Per quanto riguarda il secondo, il piano delle inchieste sul campo dell'APV, svolto da ricercatori patoisant locali formati prevalentemente all'Università di Torino e coordinato dal Centre e poi dal BREL con l'ausilio di un comitato scientifico in cui intervengono i sopracitati studiosi francesi, svizzeri e italiani, si completerà solo alla fine degli anni Novanta. Il lavoro procederà poi attraverso un laborioso (e per molti versi infruttuoso, tanto da venir in seguito abbandonato) tentativo di digitalizzazione, che determinerà di fatto un lungo stallo nell'avanzamento scientifico dell'impresa.

Dagli anni 2010, il lavoro viene finalmente ripreso attraverso una convenzione con l'Università, con l'obiettivo di procedere in direzione della pubblicazione di un primo «volume-pilota». Il decennio che separa la ripresa dell'attività con il suo compimento nel 2020 è indicativo della complessità dell'operazione, che ha contemplato lunghe e ricorsive fasi di revisione dei dati custoditi nell'archivio parzialmente informatizzato, nei quaderni d'inchiesta, nelle registrazioni delle interviste, nonché un'importante operazione di progettazione editoriale per un'opera che, arrivando dopo una così lunga attesa, non doveva tradire (per quanto possibile) le aspettative legittimamente generate nella comunità scientifica e nella comunità dei parlanti ${ }^{3}$. 


\subsection{L'APV: natura dei dati, copertura geolinguistica, dinamiche sub-areali}

Il corpus centrale delle 16 varietà francoprovenzali valdostane indagate proviene da inchieste sul campo effettuate fra il 1973 e il 1990, con un numero di informatori variabile (dai due di Saint-Oyen ai 15 di Valsavarenche), registrate e trascritte dai ricercatori all'interno di quaderni d'inchiesta annotati.

9 A questo corpus si aggiungono sei «punti di confronto» esterni, collocati nelle aree francoprovenzalofone confinanti. I materiali linguistici dei due punti svizzeri-vallesani (Liddes ed Évolène) provengono anch'essi da inchieste svolte appositamente, attraverso una convenzione con l'università di Neuchâtel, fra 1998 e 2001. Quelli dei punti piemontesi (Carema e Ribordone) sono stati integrati per gentile concessione dell'ALEPO, che ha svolto le indagini contemporaneamente all'APV. Per le due Savoie francesi, le inchieste si sono svolte negli anni Ottanta sotto la direzione di Tuaillon stesso; i dati di Les Contamines (Haute-Savoie) provengono dai quaderni d'inchiesta relativi; quelli di Tignes (Savoie), sono invece disponibili solo parzialmente nel formato di quaderni e la maggior parte di essi deriva dalla trasposizione lessicografica ospitata dall'edizione del vocabolario sulla parlata di Tignes realizzata alla fine degli anni Novanta dai ricercatori di Tuaillon (Duch \& Béyan, 1998).

10 L'omogeneità complessiva dei dati APV, sia per la parte valdostana che per i punti esterni, è comunque garantita dall'utilizzo di un comune strumento d'indagine, e cioè le più di 6.000 domande del Questionnaire pour enquêtes dialectales en pays alpin approntato originariamente da Gaston Tuaillon (1972-1973) per l'ALJA.

Figura 1. - La Valle d'Aosta e la sua copertura geolinguistica: punti d'inchiesta APV (in rosso) e degli altri atlanti (blu, ALF, celeste ALMURA, verde scuro AIS, verde chiaro ALI).

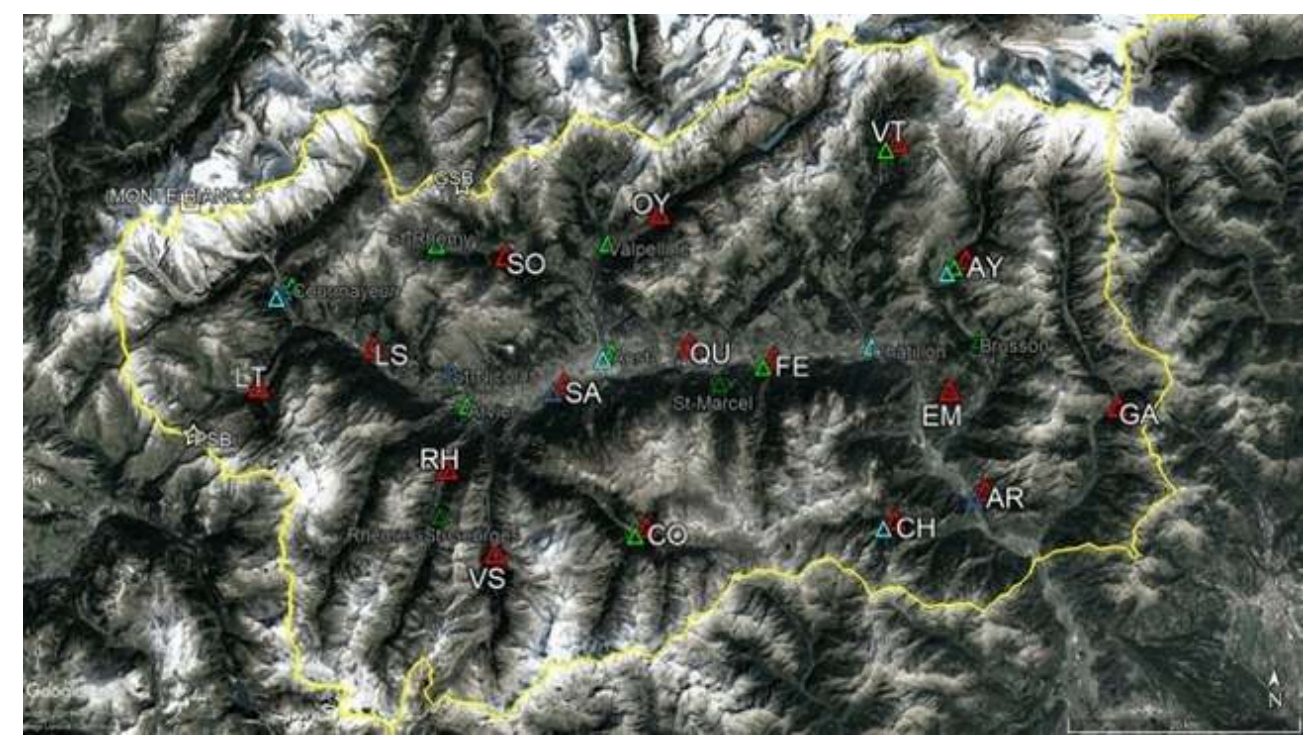

Credito: G. Raimondi / Google Earth Pro.

11 In territorio valdostano, i 16 punti d'inchiesta (fig. 1) sono stati scelti in quanto rappresentativi di altrettante sub-aree geografiche e geolinguistiche regionali ${ }^{4}$. Percorrendo da ovest ad est il corso della Dora Baltea (che nasce dal massiccio del Monte Bianco e forma la valle centrale aostana), si incontrano quindi nell'Alta Valle i 
punti di LT La Thuile (nella micro-area del Piccolo San Bernardo, sul versante destro), di LS La Salle et SA Sarre (sul fondovalle, rispettivamente a 25 e $5 \mathrm{~km}$ dal capoluogo Aosta), di SO Saint-Oyen e OY Oyace (sul versante sinistro, verso il Vallese svizzero attraverso il Gran San Bernardo o la Valpelline). Nel quadrante sud-occidentale, i punti di RH Rhêmes-Saint-Georges, VS Valsavarenche e CO Cogne testimoniano le parlate del versante destro della Dora nell'area che va dal massiccio del Rutor al Gran Paradiso e che in Francia sbocca verso l'alta valle dell'Arc e dell'Isère, in Piemonte (più a est) verso le valli Orco e Soana. A est di Aosta, il percorso che conduce allo sbocco della Valle nella Pianura Padana è punteggiato da tre punti sul fondovalle, e cioè QU Quart, FE Fénis e AR Arnad (rispettivamente a 8, 18 e $43 \mathrm{~km}$ dal capoluogo) e dal punto di EM Émarèse, posto sul versante sinistro della Dora nel retroterra montano della conca di Châtillon, dove comincia la Bassa Valle). Qui le vallate laterali sono rappresentate dai punti di VT Valtournenche e AY Ayas, per le due vallate omonime, e di GA Gaby, isola francoprovenzale all'interno della Valle del Lys germanofona (dialetti alemannici walser). Sulla destra orografica, invece, $\mathrm{CH}$ Champorcher testimonia le parlate dell'area montana meridionale racchiusa fra la valle centrale, il Mont Avic e la Val Soana piemontese.

La stessa fig. 1 riporta, a titolo di confronto, la rete di copertura geolinguistica risultante dagli atlanti nazionali preesistenti (ALF, AIS e ALI) e dall'ALMURA, atlante regionale realizzato contemporaneamente, e mostra come l'area valdostana fosse tutt'altro che scoperta. I 17 i punti indagati, alcuni dei quali esplorati da atlanti diversi (Courmayeur è presente ad esempio nell'ALF, nell'ALI e in ALMURA), rendevano già in sostanza la Valle d'Aosta una delle micro-aree maggiormente e più capillarmente coperte dalla geolinguistica italiana e francese.

13 Tuttavia, anche se la distribuzione dei punti APV (triangoli rossi) riproduce in qualche modo la distribuzione «sommativa» degli altri atlanti, il suo contributo alla comprensione delle dinamiche geolinguistiche risulta importante per diverse ragioni. Innanzitutto, la rete APV introduce la documentazione di nove punti d'inchiesta mai trattati precedentemente (La Thuile, La Salle, Rhêmes-Saint-Georges, Valsavarenche, Saint-Oyen, Oyace, Quart, Émarèse e Gaby) e mette in atto una generale operazione di riequilibrio nella distribuzione dei punti a vantaggio della parte orientale della regione (Bassa Valle), un'area particolarmente interessante dal punto di vista dialettologico (Raimondi, 2019: 27). Altre scelte di dettaglio meritano poi di essere sottolineate, per le suggestioni che offrono rispetto alla comprensione di talune dinamiche geolinguistiche specifiche dell'area, che raggrupperemo qui di seguito sottolineando da un lato le «novità» introdotte dall'APV rispetto ai predecessori, dall'altro discutendo alcune scelte relative a due «aree sensibili» nel panorama geolinguistico della regione, rappresentate dalle due conche alpine che si aprono sul fondo della valle principale della Dora, quella di Aosta e quella di Châtillon.

\subsection{La Thuile e Gaby}


Figura 2. - L'APV e la copertura geolinguistica: La Thuile e la sub-area del Piccolo San Bernardo.

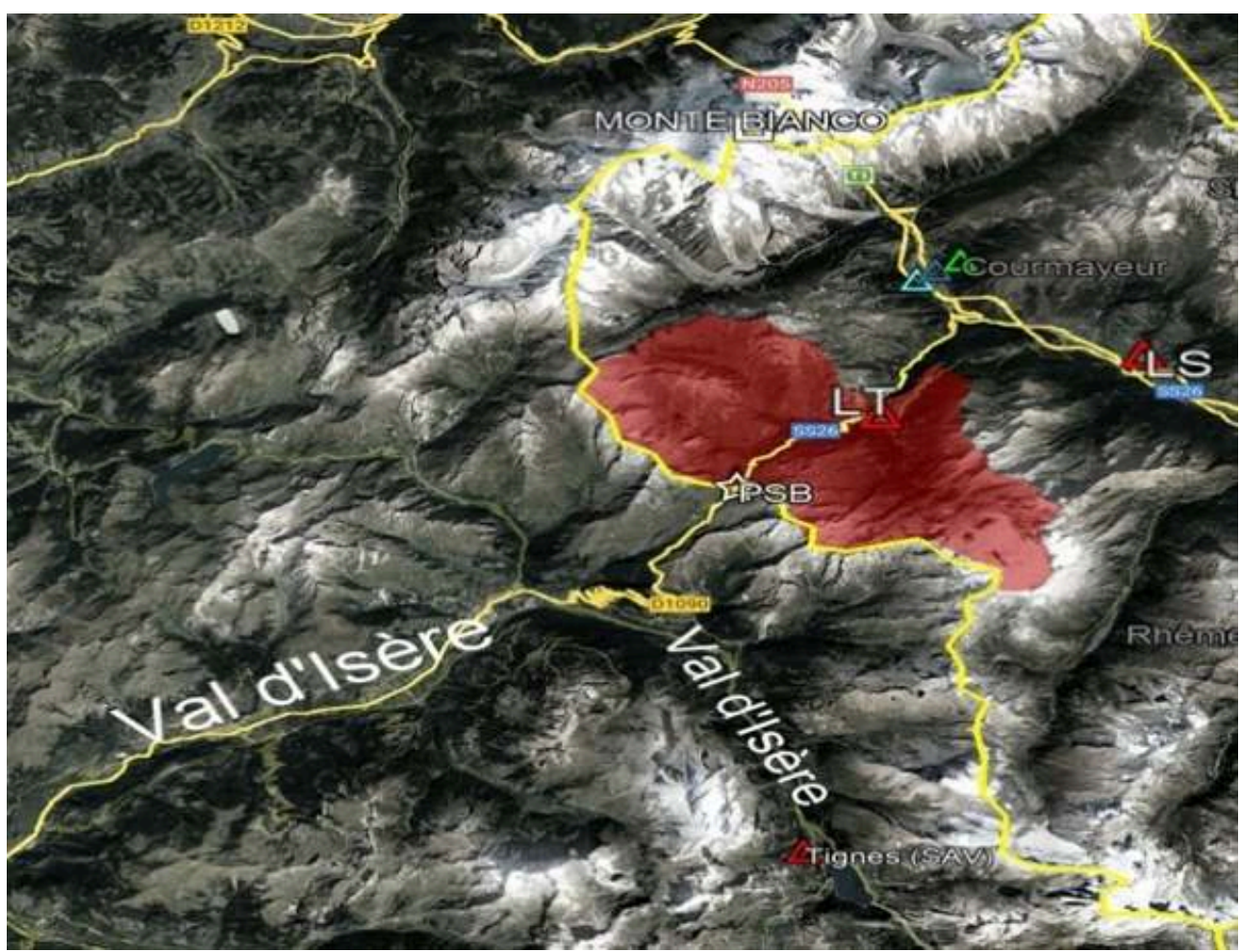

Credito: G. Raimondi / Google Earth Pro.

Fra i punti d'inchiesta introdotti per la prima volta dall'APV, spicca innanzitutto il caso di La Thuile (fig. 2). Il punto va a coprire geolinguisticamente la sub-area dialettale riferibile alla valle del Piccolo San Bernardo, che mette in comunicazione la Valle d'Aosta col retroterra della Tarentaise francese (alta valle dell'Isère, indagata nell'APV attraverso il punto esterno di Tignes) e di lì con Chambéry e con il Grenobloise. Di fatto, questa sub-area valdostana è quindi l'unica che, attraverso il valico (che in epoca medievale era la via più breve per raggiungere la capitale degli stati di Savoia), si trovava in contatto più o meno diretto con l'area francoprovenzale più meridionale in territorio francese e risulta interessante proprio per il comportamento linguistico talvolta divergente rispetto agli altri patois dell'Alta Valle, con i quali condivide comunque le caratteristiche di base (cfr. Raimondi, 2019b: 16-17, 38 e 42). 
Figura 3. - L'APV e la copertura geolinguistica: Gaby e la Valle del Lys germanofona.

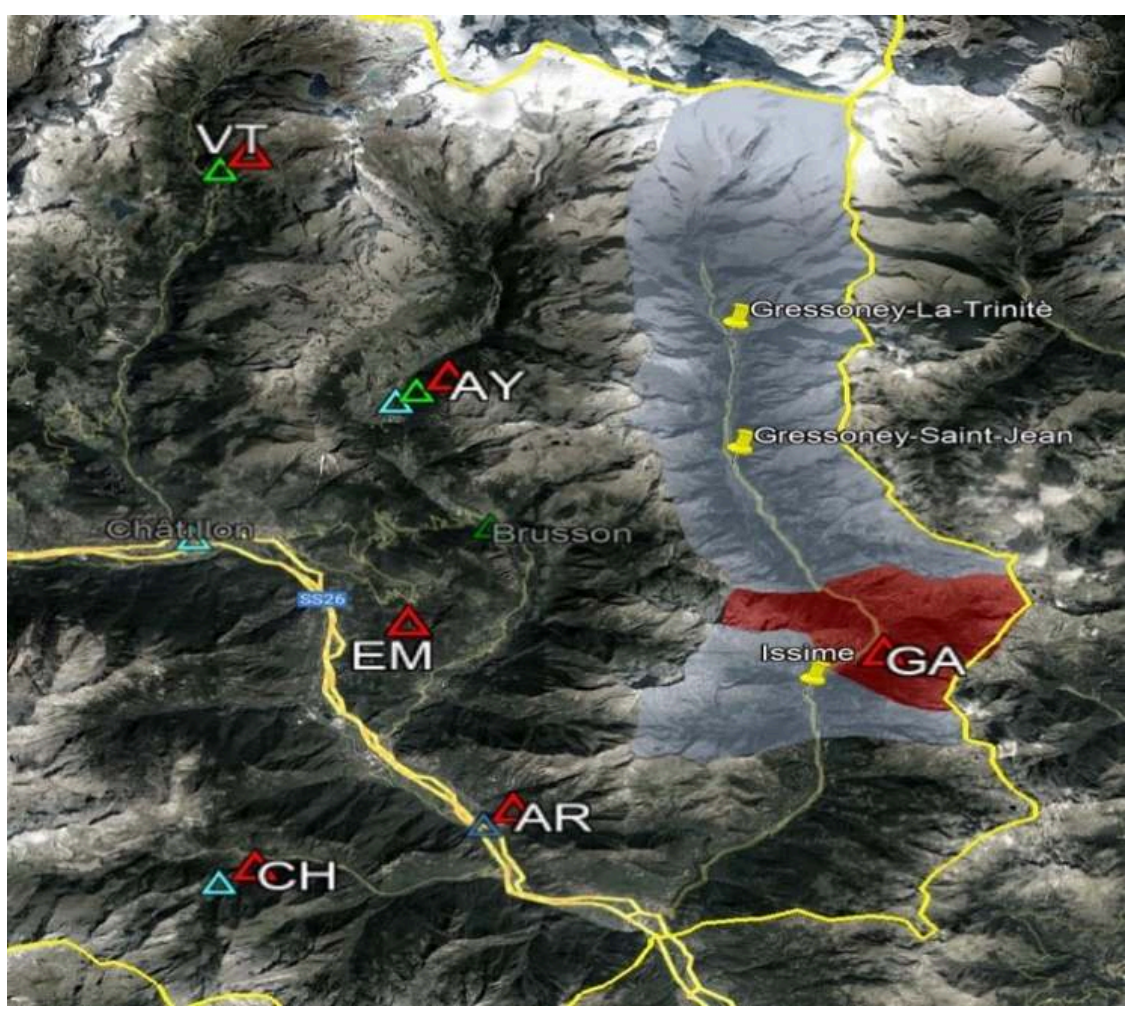

Credito: G. Raimondi / Google Earth Pro.

15 L'altro punto «nuovo» è Gaby, situato invece nella Bassa Valle (fig. 3). In questo caso, ci troviamo di fronte a un interessante e complesso caso di contatto linguistico, poiché Gaby è l'unico comune francoprovenzale in una valle (quella del torrente Lys) caratterizzata dalla presenza di una enclave germanica, e cioè dalle comunità walser dei comuni di Gressoney-Saint-Jean e Gressoney-La-Trinité (a nord di Gaby) e di Issime (a sud). Questa particolare collocazione determina per Gaby, da un lato una frequente convergenza «culturale» con i comuni germanofoni della valle, che si manifesta anche attraverso prestiti lessicali dalle loro parlate (Cioffi et al., in stampa); dall'altro, una situazione generale di distanza linguistica piuttosto pronunciata, non solo dal centro linguistico dell'area francoprovenzale valdostana (individuabile nei patois della Conca di Aosta), ma anche dalle varietà più prossime della Bassa Valle (Raimondi, 2019b: 40-42, 47).

\subsection{Aree sensibili: Conca di Aosta e Conca di Châtillon}


Figura 4. - L'APV e la copertura geolinguistica: la Conca di Aosta.

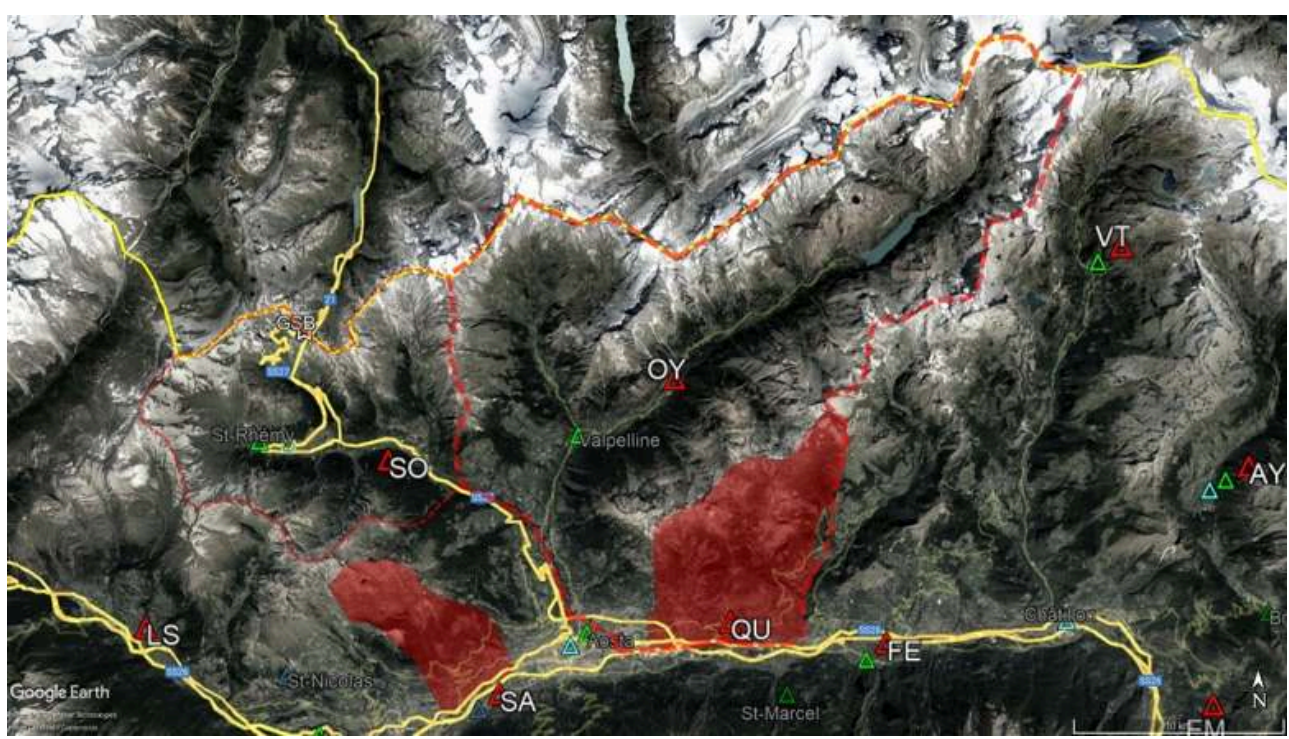

La linea rossa tratteggiata indica i confini variabili della castellania medievale di Quart.

Credito: G. Raimondi / Google Earth Pro.

16 Altre configurazioni di copertura proposte dall'APV meritano una discussione supplementare. È ad esempio il caso del capoluogo regionale Aosta, punto d'inchiesta di ALI e ALMURA ma non nel nostro atlante. L'APV propone a questo riguardo una copertura «a due fuochi» dell'area più urbanizzata della regione, scegliendo i due comuni rurali che delimitano la cosiddetta "Conca di Aosta» (l'area relativamente pianeggiante frutto dell'incontro fra il ghiacciaio del Monte Bianco e quello del Grand-Combin, dove, alla confluenza fra la Dora e il Buthier, fu fondata nel 25 a.C. la Aosta romana): ad ovest (SA Sarre) e ad est (QU Quart; cfr. fig. 4). Si dovrà qui notare come, in un certo senso, questa scelta si configuri già come una sorta di «ipotesi di lavoro» dialettologica, considerando Aosta più che un'area urbana (peraltro già indagata dai due atlanti nazionali ALF e ALI) come punto di confluenza di due sub-aree linguistiche, orientate la prima verso l'Alta Valle, la seconda verso l'area mediana, in particolare quella che si estende sul versante sinistro della Dora in direzione del Gran San Bernardo 5 .

17 Un secondo e forse più delicato tema è poi rappresentato dall'indagine della fascia intermedia di transizione fra il sottogruppo dei patois dell'Alta e della Bassa Valle, che (come abbiamo anticipato: cfr. sopra nota 4) costituisce la principale partizione dialettale del francoprovenzale valdostano e che forse avrebbe richiesto maggiore attenzione di dettaglio nella fase iniziale della scelta dei punti d'inchiesta. Se infatti i punti estremi delle isoglosse lessicali e fonetiche nord-est/sud-ovest che tagliano la regione risultano sufficientemente individuati dalla presenza nella rete APV delle località di VT Valtournenche e AY Ayas a nord (dove il punto d'attacco oscilla si colloca fra le due valli confinanti, a volte includendo, a volte escludendo la prima dall'area della Bassa Valle), di CO Cogne e CH Champorcher (apparentemente distanti, ma in realtà circondate da un'ampia area in altura e disabitata fra massiccio del Gran Paradiso e Mont Avic), è invece l'area di fondovalle che circonda la Conca di Châtillon a risultare debolmente definita. 
18 All'epoca, la scelta di EM Émarèse come unico punto ubbidì a comprensibili logiche di complementarità dell'informazione dell'APV rispetto agli atlanti preesistenti, dato che ad esempio Châtillon (situata ad ovest del contrafforte montano che chiude a sud-est la Conca, la cosiddetta «Chiusa di Monjovet») era già documentata dall'ALF. Tuttavia, considerando invece come prioritario il quadro informativo «interno» all'APV, un supplemento di indagine che mettesse in luce la dialettica variazionale che intercorre spesso fra le località situate ad est (come appunto Émarèse) o ad ovest della Chiusa, sarebbe forse stato opportuno e meriterebbe ancora oggi un approfondimento supplementare. Come si è potuto altrove mettere in luce (cfr. Raimondi, 2014), è infatti proprio in quest'area di fondovalle che le isoglosse sopramenzionate si incrociano, con gli andamenti spesso differenziati evidenziati in fig. $5^{6}$.

Figura 5. - L'APV e la copertura geolinguistica: l'area intermedia fra patois dell'Alta e della Bassa Valle.

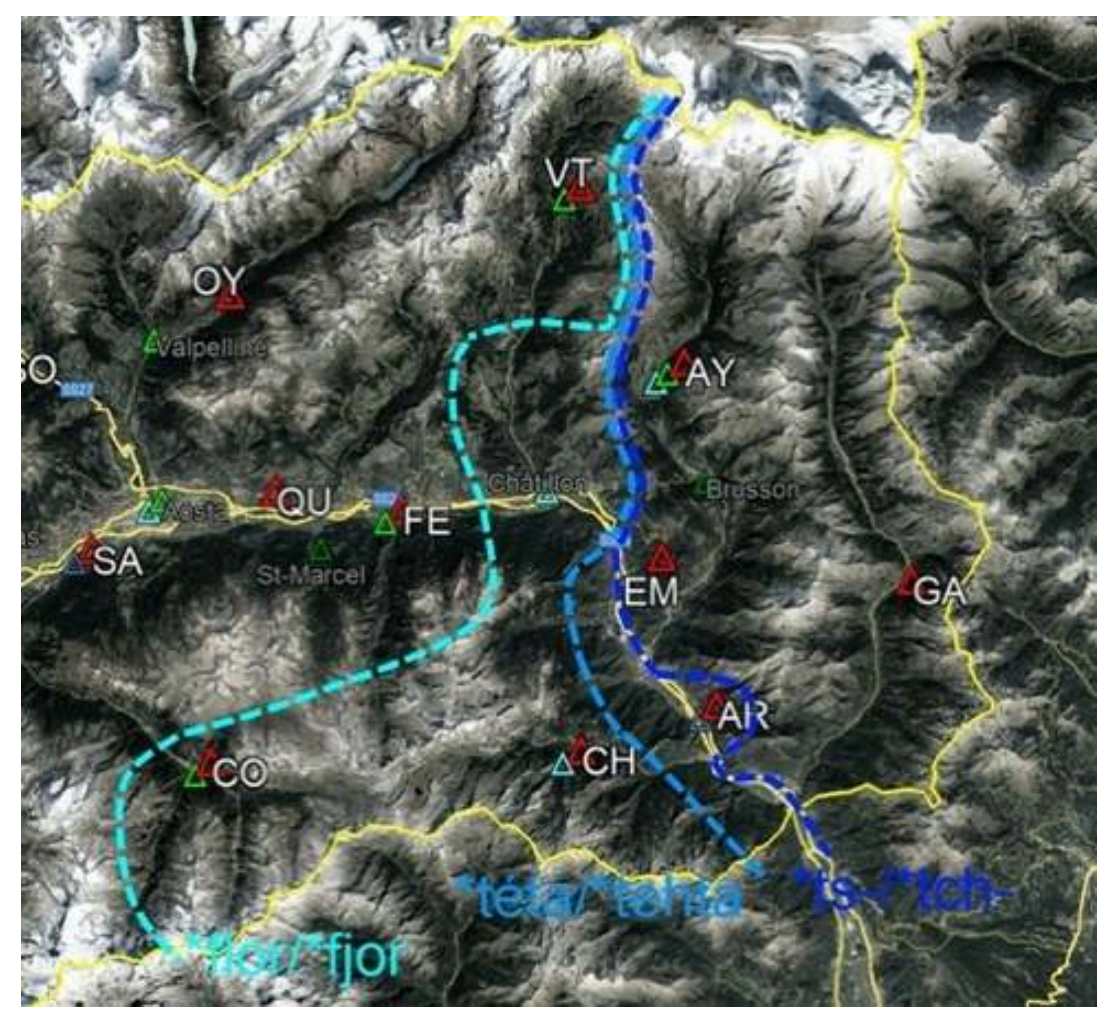

Le linee tratteggiate riportano tre isoglosse fonologiche (da Raimondi, 2014: 783).

Credito: G. Raimondi. / Google Earth Pro.

\section{APV/1: Le lait et les activités laitières}

19 La lunga gestazione della fase di edizione dell'APV è stata anche determinata dalla volontà di produrre un'opera scientifica all'altezza delle aspettative, conseguenti alla lunga attesa. Accanto quindi alle operazioni di controllo del dato, di revisione e di correzione (ben note a tutti gli studiosi che si occupino di geolinguistica e di atlanti), una cospicua dose delle energie redazionali è stata dedicata alla progettazione del prodotto editoriale in sé, con l'obiettivo di raggiungere un bilanciamento fra tre aspetti: l'accessibilità alla ricca mole di dati contenuta nelle inchieste, la leggibilità dei 
suoi contenuti anche per un utente non specialistico, la presenza di aspetti di originalità metodologica e scientifica. Nei temi che illustreremo sotto, tutti e tre gli aspetti sono presenti, anche se naturalmente con pesi e modalità differenti.

\subsection{L'articolazione referenziale dell'APV}

Una parte molto rilevante del lavoro redazionale dell'APV in vista della pubblicazione è rappresentata dalle operazioni di strutturazione dell'insieme dei dati ricavati dalle inchieste in unità informative dotate di un'intitolazione (Articles, nel metalinguaggio dell'atlante), composte primariamente da carte analitiche o raccolte di brevi testi (a seconda dei casi) e da un apparato di note e varianti (cfr. Raimondi, 2019b: 8; Raimondi 2019a, per i dettagli).

Figura 6. - Un estratto della Table des matières.

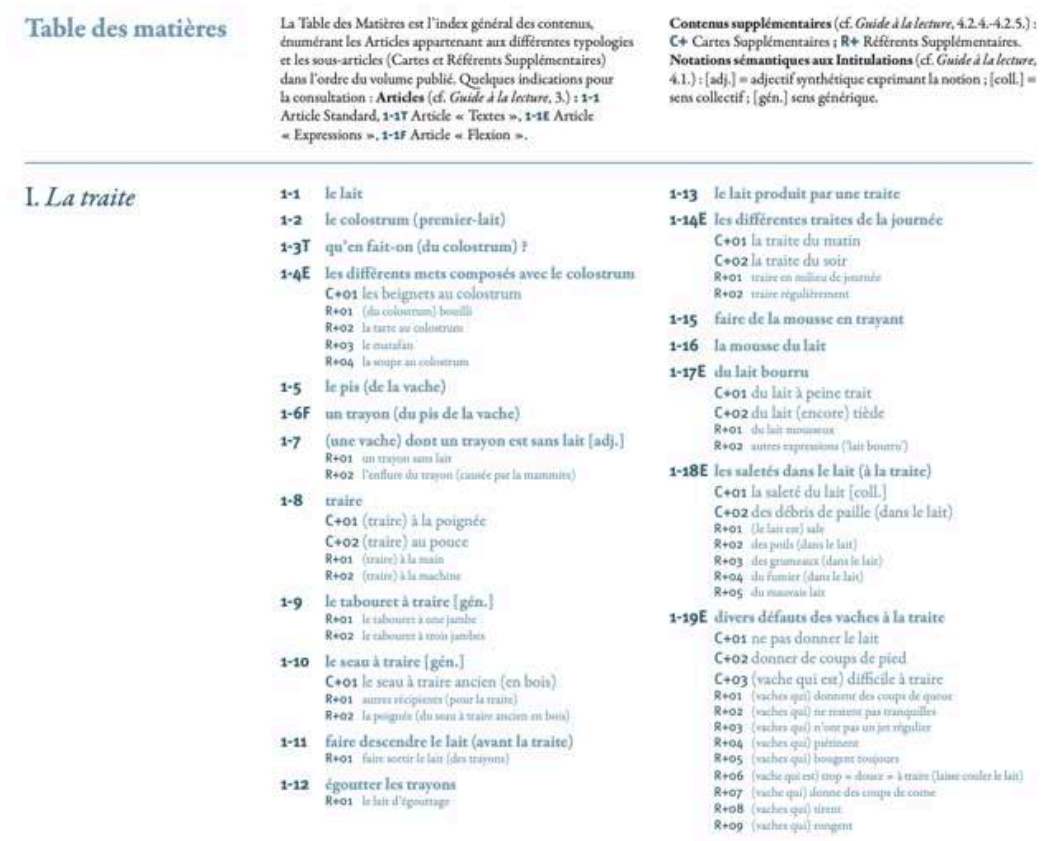

Credito: G. Raimondi.

21 Come si può notare nell'estratto di indice in fig. 6, le voci APV hanno spessissimo una struttura articolata, con contenuti principali (nell'esempio il verbo traire 'mungere') e contenuti supplementari o nozioni aggiuntive, che derivano dallo spoglio accurato dei quaderni d'inchiesta e delle registrazioni delle interviste originarie, durante le quali le dinamiche interazionali generate dalla natura «semidirettiva» del questionario Tuaillon hanno sovente prodotto flussi informativi che vanno ben al di là della diade «domanda/risposta». 
Figura 7. - Un esempio del trattamento dell'informazione (APV/1, 2020: 36-38), dal quaderno d'inchiesta (Valsavarenche), alla strutturazione dell'article (APV/1-8 traire), ai Référents supplémentaires proposti in forma di tabella $(\mathrm{R}+)$ e alle due Cartes supplémentaires $(\mathrm{C}+)$ per le nozioni di (traire) à la poignée e (traire) au pouce.

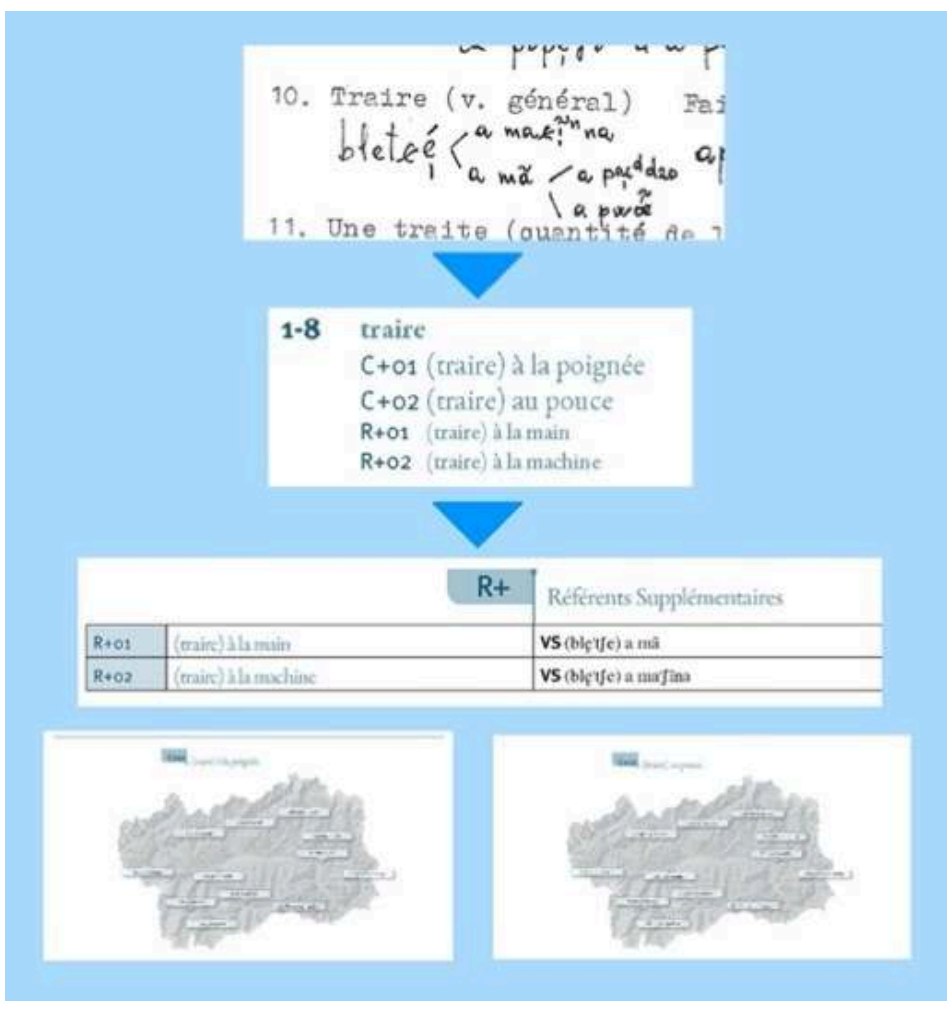

Credito: G. Raimondi.

L'articolazione dell'APV deriva quindi dalla riconduzione di questo flusso spontaneo di informazioni ad altrettanti referenti esplicitabili attraverso un'intitolazione (cfr. fig. 7), i quali vanno a formare nuove unità informative secondarie (Référents supplémentaires, indicati nelle intitolazioni come $R+$ ) e che arricchiscono notevolmente la griglia referenziale dell'atlante.

Per dare un'idea della ricchezza informativa prodotta, a partire dalle 126 domande originarie del questionario utilizzate per questo volume si sono ottenuti 112 Articles e ben 188 voci secondarie, per un totale di 300. Una buona parte di queste nozioni supplementari (37), in particolare quelle ricorrenti in almeno sette punti d'inchiesta, sono poi stati anche cartografate, producendo le Cartes supplémentaires ( $C_{+}$; cfr. nuovamente fig. 7). Per questa operazione (che ha permesso anche l'indicizzazione analitica di tutte le nozioni supplementari; v. oltre), l'APV è sicuramente debitore del concetto di «questionario a posteriori», elaborato da Tullio Telmon e Sabina Canobbio (1994:33) nel cantiere dell'ALEPO per rispondere a una delle massime auree della ricerca sul campo in dialettologia, secondo la quale «si scopre come avrebbe dovuto essere fatto un questionario solo dopo aver ottenuto le risposte degli informatori».

\subsection{Glossari, indici, repertori}

La «vocazione didattica» dell'APV (che nasce anche come segno tangibile di una restituzione dei frutti della ricerca dialettologica alla comunità dei parlanti) si manifesta attraverso l'ampio spazio lasciato ai commentari esplicativi (referenziali, 
linguistici ed etnografici) che introducono ciascuna delle voci principali (cfr. Raimondi, 2019b: 9) ma anche attraverso una particolare ricchezza di strumenti di accesso all'informazione o di corredo ad essa.

Fra questi, uno strumento particolarmente attento ai bisogni del sopracitato «lettore medio» non specialista è innanzitutto rappresentato dal Petit glossaire des termes de linguistique utilisés dans l'Atlas (APV/1, 2020: 15-17), che spiega e commenta con esempi circa 130 lemmi terminologici, da Affriquée (consonne) a Walser (dialectes) (fig. 8).

Figura 8. - Un estratto del Petit glossaire des termes de linguistique utilisés dans l'Atlas (APV/1, 2020: 15).

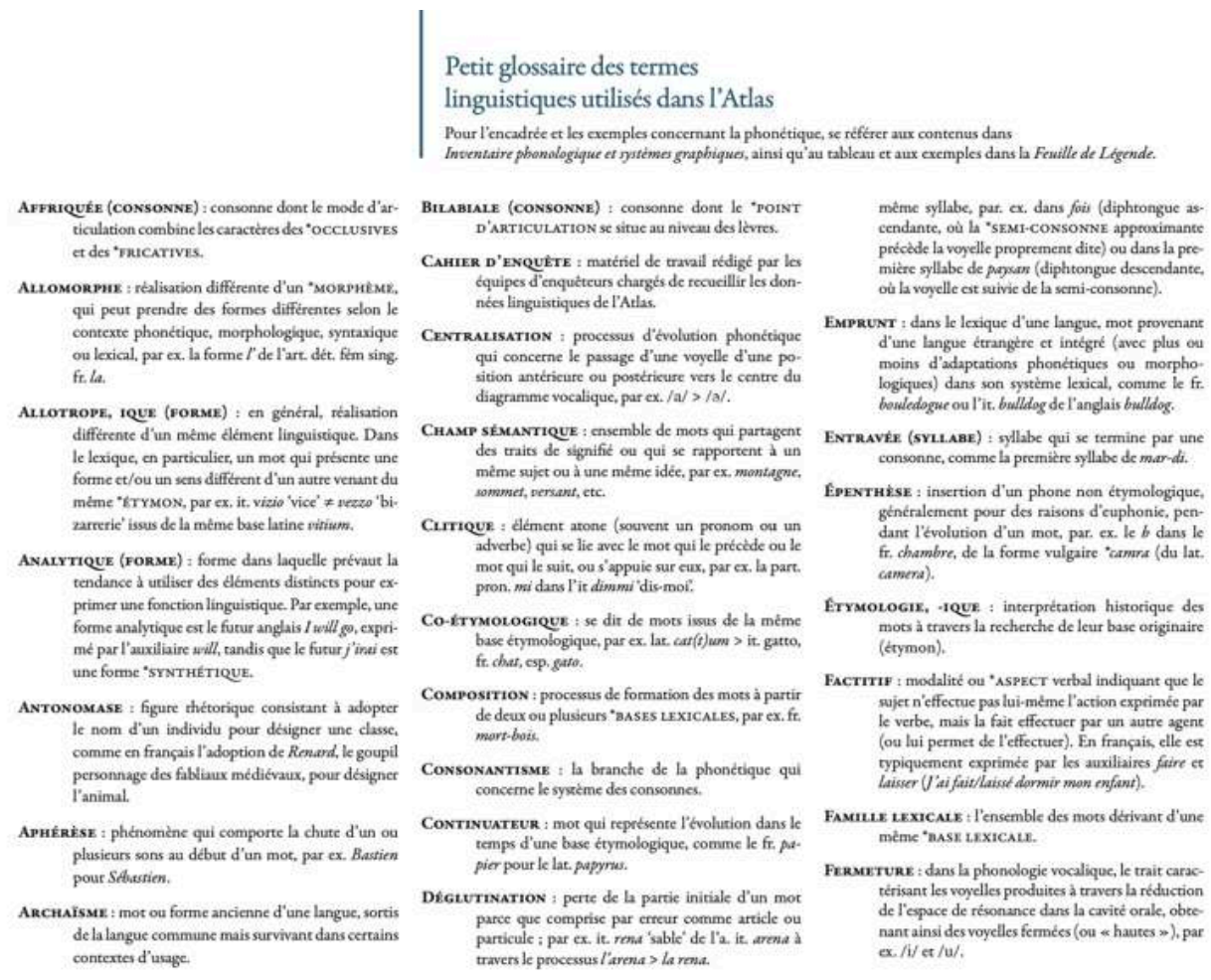

Credito: G. Raimondi.

La consultabilità trasversale dei dati dell'atlante si appoggia invece su tre indici, che permettono al lettore l'accesso a partire tanto dal lato del «referente» che da quello del «significante». Per il primo aspetto, il paziente lavoro di articolazione dei contenuti cui si accennava sopra determina una notevole ricchezza di accesso. Accanto alla Table des matières già mostrata (che visualizza sinotticamente l'intitolazione della voce principale ma anche quelle dei referenti supplementari in cui la voce si articola), l'accesso completo ai referenti tanto primari che secondari è infatti garantito anche da un'indicizzazione analitica particolarmente approfondita (Index analytique français, APV/1, 2020: 210-215, cfr. fig. 9), in funzione della quale sono state spogliate tutte le intitolazioni primarie e secondarie degli Articles, estraendo da esse tutte le occorrenze verbali, sia per i lessemi autosemantici (nomi, verbi, aggettivi, avverbi), sia per quelli sinsemantici. Le occorrenze sono state poi lemmatizzate secondo la prassi lessicografica nei Lemmes Grammaticaux; un secondo tipo di rimando è poi rappresentato dai Lemmes Thématiques (in maiuscoletto nell'esempio), creati qualora le 
occorrenze suggerissero un richiamo indiretto all'oggetto o al concetto, non necessariamente collegato a una possibile «traduzione» nelle risposte.

Figura 9. - L'Index analytique français (APV/1, 2020: 210-215).

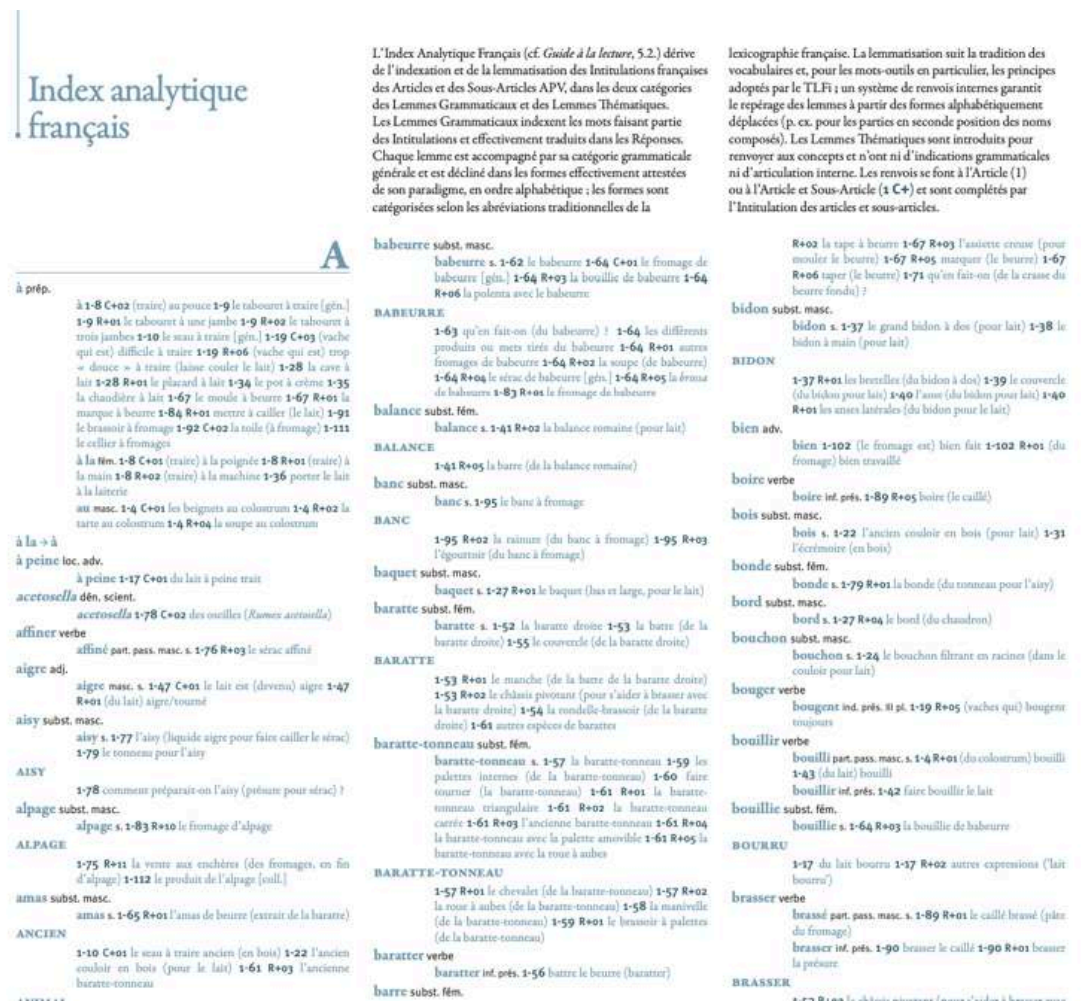

Credito: G. Raimondi. 
Figura 10. - L'Index des types lexicaux patois (APV/1, 2020: 216-223).

Index des types

lexicaux patois
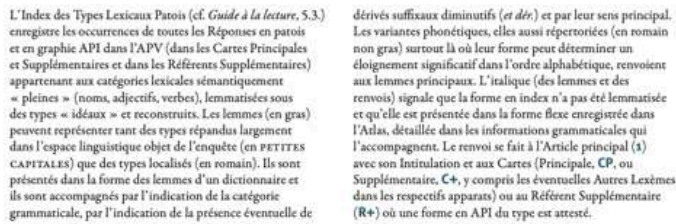

A

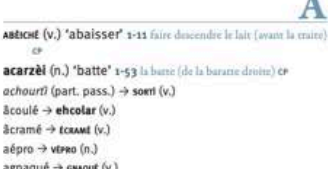

agota $\rightarrow$ teomt $(v)$

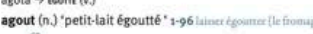

agoutchi $\rightarrow$ teont (vi)

suete $\rightarrow \rightarrow$ cutrt $(v)$

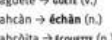

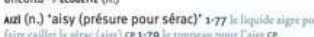

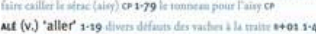

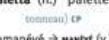

amanéret $\rightarrow$ muxort $(1)$

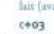

ancro (adi). 'rance' 1.-51 ll beurre) ar anceo

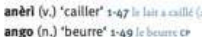
ango (n.) "beurre' 1.49 ) anss (n) et dér. 'anse.

Credito: G. Raimondi.
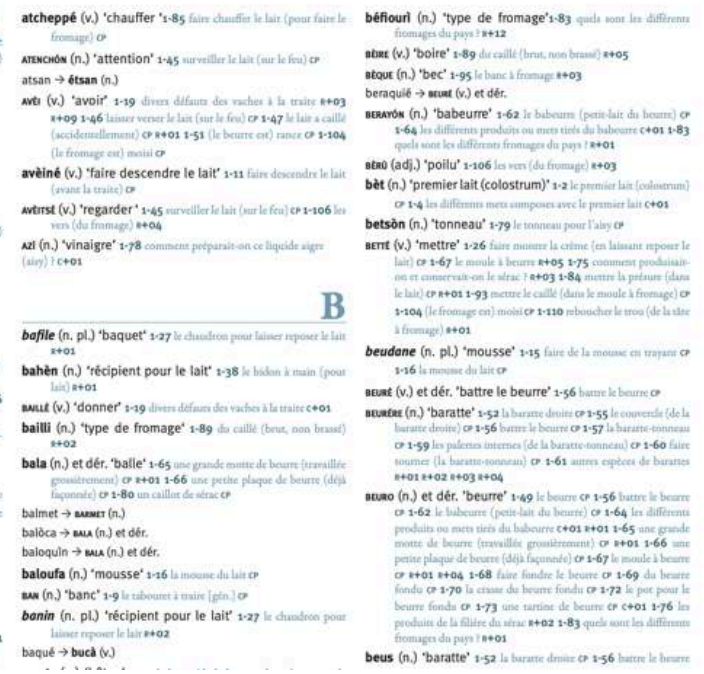
types lexicaux patois che correda l'APV (APV/1, 2020: 216-223, cfr. fig. 10) raccoglie le occorrenze di tutti i lessemi dialettali presenti nelle risposte e appartenenti alle categorie grammaticali di nomi, verbi e aggettivi. La lemmatizzazione avviene attraverso la riconduzione delle occorrenze a lemmi dialettali ideali trascritti in grafia alfabetica (in maiuscoletto grassetto nell'esempio) ed espressi secondo la forma del patois centrale valdostano (quello della Conca di Aosta), con conseguente riduzione ad essa delle varianti fonetiche $e^{7}$. Quando il tipo lessicale sia invece esclusivamente periferico (e questo accade abbastanza spesso, ad esempio per i lessemi presenti solo nei patois della Bassa Valle o per quelli dei punti esterni) esso viene portato a lemma (in minuscolo grassetto) nella forma linguistica più frequente o etimologicamente più «trasparente» attestata nell'atlante. L'utilizzo di rimandi $(\rightarrow)$ permette poi il reperimento anche delle forme foneticamente divergenti rispetto a quella portata a lemma, anch'esse regolarmente censite e registrate nella posizione alfabetica pertinente.

Ai contenuti maggiormente specialistici sono invece dedicati una serie di Répertoires. Tali contenuti provengono dall'elaborazione interpretativa compiuta dalla redazione nella fase di costruzione e di commento delle voci e di realizzazione delle cartine sintetiche (su cui cfr. Raimondi, 2019b: 8-10, 14-24), approntate in numero decisamente elevato (393) per rendere leggibili con più immediatezza le configurazioni geolinguistiche emergenti. Gli elementi linguisticamente o etnograficamente rilevanti tratti da commenti e cartine sintetiche sono stati quindi spogliati dando luogo a quattro repertori (fig. 11): 1. il Répertoire lexical, il più corposo (APV/1, 2020: 224-238), che ospita lo spoglio di tutti la terminologia di interesse lessicale presente (etimi, 
termini in altre lingue citati per confronto, significati, «iconimi», per cui cfr. oltre); 2. il Répertoire phonétique, che presenta sia un Index par phénomènes (articolato secondo tradizione in vocalismo, consonantismo e altri fenomeni, e ordinato a partire dal fonema originario latino), che un Index par phones (ordinato invece sulla base dei foni risultanti dall'evoluzione fonetica); il 3. Répertoire morpho-syntaxique e il 4. Répertoire ethnographique, che presentano invece semplicemente le liste delle carte di sintesi dedicate ai due ordini di aspetti.

Figura 11. - I Répertoires: 1. Lexical (APV/1, 2020: 224-238), 2. Phonétique (APV/1, 2020: 239-242), 3. Morphosyntaxique e 4. Ethnographique (APV/1, 2020: 224).

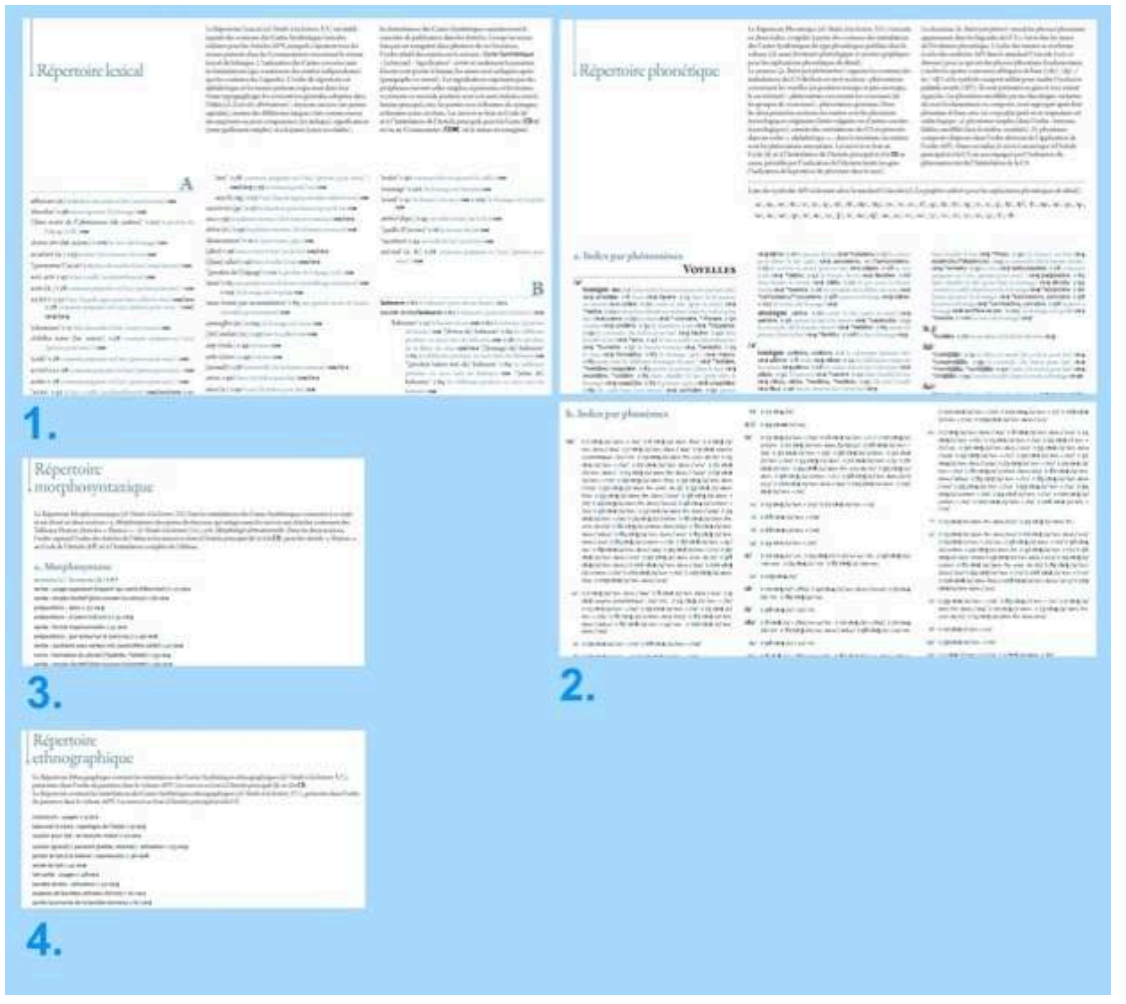

Credito: G. Raimondi.

\subsection{Lo strumento della «motivazione» nell'APV}

Tracciato nelle sue linee essenziali dalle riflessioni di Stephen Ullmann negli anni Cinquanta del Novecento, ma poi sviluppato compiutamente in una complessiva «teoria della motivazione» da Mario Alinei negli anni Novanta, lo strumento concettuale della motivazione si è affermato nel tempo come uno dei più innovativi nel campo dell'analisi semantica della lingua e della lessicografia, tanto da informare di sé entrambi i più importanti progetti di atlanti linguistici sovranazionali delle lingue europee, l'ALiR - Atlas linguistique roman e l'ALE - Atlas Linguarum Europae. Intendendo il concetto di motivazione secondo i canoni (di stretta osservanza alineiana; cfr. Alinei, 2009: 70-88 per la loro formulazione più aggiornata) che lo vedono direttamente chiamato in causa nel processo di lessicalizzazione (ovvero il momento della «genesi del significato» di un nuovo lessema), nell'APV l'approccio è utilizzato in maniera piuttosto sistematica nella cartografia sintetica, utilizzando tanto la terminologia (come per il calco francese iconyme basato sulla coniazione iconimo), quanto la 
simbologia (l'uso delle parentesi graffe per gli iconimi), quanto la complessiva architettura concettuale proposta da Alinei, che distingue ad esempio «iconimi semplici» e «classi di iconimi» 8 .

Sono venti le carte sintetiche lessicali di tipo motivazionale, marcate esplicitamente LEX-M nel metalinguaggio editoriale dell'atlante (accanto a quelle tradizionalmente onomasiologiche LEX e a quelle semasiologiche LEX-S). Operativamente, nell'APV l'utilizzo di questo strumento è risultato funzionale per gestire due differenti ordini di problemi. Secondo la prospettiva più classica adottata anche da ALiR e ALE, l'utilizzo dello strumento interpretativo della motivazione ha aiutato a risolvere, sul piano della rappresentazione geolinguistica, $\mathrm{i}$ casi in cui l'abbondanza e la dispersione di significanti nasconde in realtà quadri motivazionali più semplici e orientati, risalendo appunto a quello che Alinei chiamava «il cordone ombelicale» che lega la parola all'atto tassonomico cognitivo che la precede, cioè all'iconimo. Nella fig. 12, la carta sintetica motivazionale sulla sinistra (CS-2 tratta dalla voce APV 1-13 le lait produit par une traite; APV/1, 2020: 45) mostra ad esempio la convergenza motivazionale verso l'iconimo \{traire\} 'mungere' (rappresentato dal triangolo blu) delle denominazioni etimologicamente differenziate *mout (deverbale da *moudre < lat. *MULGĔRE, diffuso nella Bassa Valle) e *ariaye (derivato da *arié < lat. tardo *arredare 'curare (le bestie)' e poi 'mungere', formato sulla base germ. ${ }^{*}$ RĒPS 'beni, provviste', nel punto vallesano di Évolène). Sulla destra, nella carta sintetica tratta dalla voce APV 1-53 la batte (de la baratte droite) (APV/1, 2020: 104) sono ben cinque $\left(1 .{ }^{*}\right.$ moda, *modòn < lat. *MOVITARE; 2. vèira < lat. VIRARE; 3. foillòn < lat. FULLARE; 4. piston < lat. PISTARE; 5. acarzèy < lat. *aDCALC(E)ARE) le denominazioni deverbali riconducibili all'iconimo sovraordinato \{fonction\} che si oppongono (anche geolinguisticamente) a quelle del tipo *bâton o baquièt ( $<$ lat. tardo *BACCU), motivate invece analogicamente (\{forme\}) e diffuse nella parte centrale del dominio valdostano e a quella periferica ruéla 'rotella', ispirata alla parte terminale rotonda del bastone della zangola da burro verticale.

Figura 12. - Esempi di carte sintetiche lessicali-motivazionali.

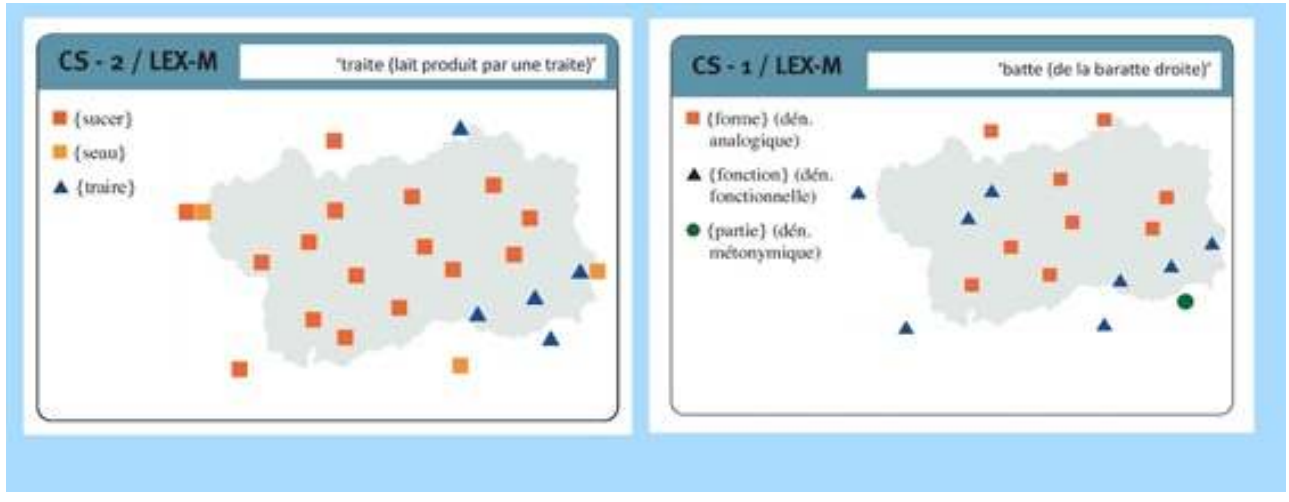

Credito: G. Raimondi.

31 In altri casi, la classificazione motivazionale è stata utile, se non a ricostruire una coerenza geolinguistica, almeno ad inquadrare sinteticamente i paradigmi di possibilità espressive per gli stimoli del questionario che hanno prodotto risposte traduttive, descrittive o comunque «sfocate» nel rapporto fra referente proposto e denominazioni ottenute. L'uso delle sintesi motivazionali in questi casi, da un lato esplicita al lettore il carattere problematico della nozione nell'enciclopedia del parlante; dall'altro permette 
alla redazione di rispettare e processare lo stato originario dei dati, evitando processi metodologicamente non lineari, come sarebbe l'attingere a fonti diverse per «riempire i buchi», siano pure esse forme supplementari di inchiesta, ripetute però nel nostro caso a cinquant'anni di distanza da quelle originali.

Negli esempi in fig. 13, tanto le denominazioni metonimiche per il filtro dell'imbuto da latte (a sinistra, tratto da APV 1-24 le bouchon-filtrant (du couloir); cfr. APV/1, 2020: 64-65) ${ }^{9}$, quanto quelle sinonimiche o perifrastiche per 'sorvegliare (il latte)' (a destra, APV 1-45 surveiller le lait; cfr. APV/1, 2020: 93) ${ }^{10}$, sono state trattate tutte come risposte «valide» allo stimolo proposto, in quanto rappresentative della dinamica stimolo-risposta prodottasi al momento dell'inchiesta. A questo titolo, esse sono state mantenute nella documentazione e cartografate simbolicamente in un quadro di tipo motivazionale che opporrà quindi un certo numero di risposte sintetiche, senz'altro più soddisfacenti in rapporto alle attese del ricercatore, ad altre meno soddisfacenti ma comunque rispondenti ad una qualche strategia di risposta messa in atto dai testimoni.

Figura 13. - Esempi di carte sintetiche lessicali-motivazionali.

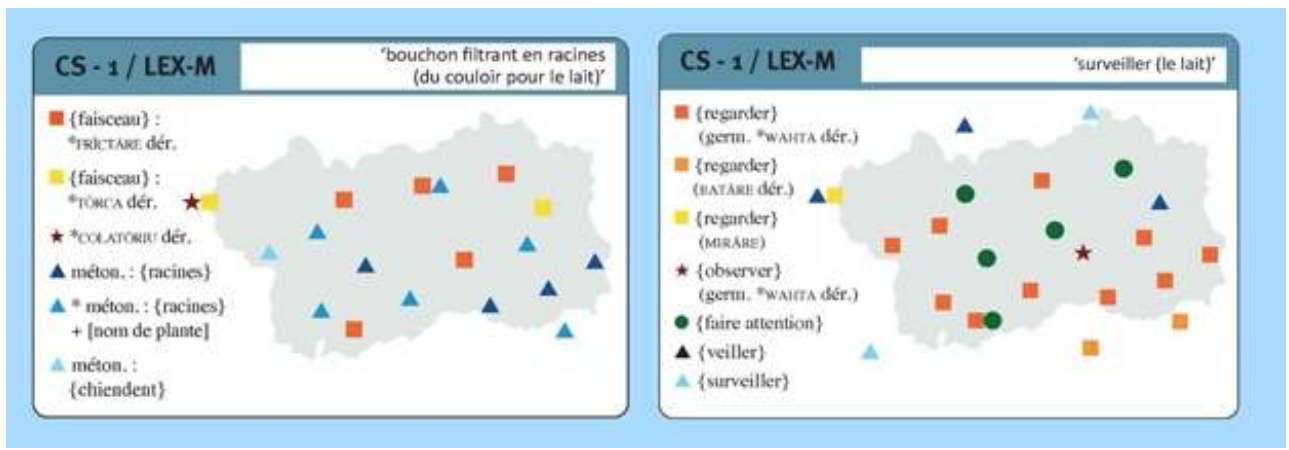

Credito: G. Raimondi.

\section{Conclusioni}

Da quanto esposto finora emergono implicitamente alcuni dei principi serviti da guida durante la progettazione della veste editoriale definitiva dell'atlante.

Uno di questi principi è stato senz'altro il «rispetto del dato». Per quanto sia evidente per chiunque lavori a partire da inchieste sul terreno la natura imperfetta, a volte manchevole, dei dati così ottenuti, nel caso specifico dell'APV si è deciso da subito di basarsi per la pubblicazione esclusivamente sui dati ottenuti dalle inchieste originali, senza ricorrere cioè ad ulteriori inchieste per colmare gli eventuali vuoti da questo punto di vista, è prevalsa insomma l'idea (che crediamo scientificamente corretta) di considerare i materiali d'archivio come un corpus, comunque rappresentativo di un particolare «stato di lingua» (quello del patois valdostano negli anni Settanta del secolo trascorso), di cui rispettare l'integrità.

Lo sforzo progettuale è stato quindi indirizzato nella direzione di uno sfruttamento minuzioso della ricchezza di informazioni (linguistiche, ma anche antropologiche e culturali) comunque disponibile. Questo sfruttamento è stato perseguito attraverso un lavoro estremamente accurato di preedizione, da cui è scaturita ad esempio la struttura ricca ed estremamente articolata della griglia referenziale, ma anche dall'attenzione agli apparati di accesso a questa informazione, che crediamo l'esposizione di queste 
pagine abbia messo in evidenza. A questo lavoro tipicamente redazionale, l'APV ha cercato comunque di abbinare il contributo di una riflessione scientifica sui metodi di interpretazione che un approccio linguistico moderno deve saper applicare anche a materiali come quelli prodotti dalle inchieste realizzate cinquant'anni or sono, realizzate nel solco di una dialettologia assolutamente «tradizionale»; nel senso naturalmente migliore che si possa attribuire a questo aggettivo. Sotto questo rispetto, l'approccio motivazionale, con gli addentellati che esso offre con campi assolutamente ancora in via di esplorazione come la semantica cognitiva di stampo lakoffiano, può rappresentare un terreno sicuramente ancora fecondo per esplorare la ricchezza di quei meravigliosi universi dell'espressione linguistica che sono i dialetti.

\section{BIBLIOGRAFIA}

AIS = JABERG Karl \& JUD Jakob, 1928-1940, Sprach-und Sachatlas Italiens und der Südschweiz, Zofingen, Ringier.

ALEPO = Atlante Linguistico ed Etnografico del Piemonte Occidentale, Università degli Studi di Torino, Dipartimento di Studi Umanistici, diretto da Riccardo Regis (<www.alepo.eu>, anche per la lista dei volumi editi).

ALE = Atlas Linguarum Europae, 1983-1990, Assen-Maastricht (voll. 1-4) \& 1997-2008, Roma, Istituto Poligrafico e Zecca dello Stato (voll. 5-7).

ALF = GILLIÉRON Jules \& EDMONT Edmond, 1902-1910, Atlas linguistique de la France, Paris, Champion.

ALI = Atlante Linguistico Italiano, Università degli Studi di Torino, Dipartimento di Studi Umanistici, diretto da Matteo Rivoira (<www.atlantelinguistico.it/>, anche per la lista dei volumi editi).

ALINEI Mario, 2009, L'origine delle parole, Roma, Aracne.

ALiR = Atlas linguistique roman, 1986-..., Roma, Istituto Poligrafico e Zecca dello Stato (voll. $1 \mathrm{e}$ 2a-b) e Alessandria, Edizioni dell'Orso (vol. 2c).

ALJA = MARTIN Jean-Baptiste \& TUAILLON Gaston, 1971-1978, Atlas linguistique et ethnographique du Jura et des Alpes du Nord, Paris, CNRS.

ALMURA = Atlas linguistique multimédia de la région Rhône-Alpes. Consultabile online su $<\mathrm{https}: / /$ www.atlas-almura.net/>.

APV/1 2020: FAVRE Saverio \& RAIMONDI Gianmario (a cura di), 2020, Atlas des patois valdôtains / 1: Le lait et les activités laitières, Aosta, Le Château.

CANOBBio Sabina \& Telmon Tullio, 1994, Atlante Linguistico e Etnografico del Piemonte occidentale. Questionario. I Introduzione, Torino, Regione Piemonte.

CHENAL Aimé \& VAUTHERIN Raymond, 1997, Nouveau dictionnaire de patois valdôtain, Aosta, Musumeci. 
Cioffi Raffaele, Angster Marco, Bellante Marco, Benedetto Mas Paolo, GaEtA Livio, Murelli Adriano, Pons Aline, RAIMONDI Gianmario \& RIVOIRA Matteo, in stampa, «Mountain linguistics: The Western Alpine Landscape», in 54th Annual Meeting of the Societas Linguistica Europaea (SLE 2021), proceedings of the online conference, 30 August-3 September 2021 (WS 17: Mountain Linguistics).

Duch Célestin \& BÉfAN Henry, 1998, Le patois de Tigne (Savoie), Grenoble, ELLUG.

Index zum AIS = JABERG Karl \& JUD Jakob, 1960, Index zum Sprach-und Sachatlas Italiens und der Südschweiz. Ein propädeutisches etymologisches Wörterbuch der italienischen Mundarten, Bern, Stämpfli \& Cie.

KELLER Hans-Erich, 1958, Études linguistiques sur les parlers valdôtains, Bern, A. Francke.

Nichols Johanna, 2015, «Types of spread zones: Open and closed, horizontal and vertical», in R. de Busser and R. J. LaPolla (a cura di), Language structure and environment: Social, cultural, and natural factors, Amsterdam / Philadelphia, J. Benjamins, p. 261-286.

RAIMONDI Gianmario, 2012, I nomi di persona in Valle d'Aosta fra XVI $I^{e} \mathrm{VII}^{e}$ secolo. Interferenze linguistiche, interferenze culturali, Aosta, Le Château.

RAIMONDI Gianmario, 2019a, «APV-Atlas des patois valdôtains», in T. Krefeld e R. Bauer (a cura di), Lo spazio comunicativo dell'Italia e delle varietà italiane. Korpus im Text. Consultabile online su <https:// www.kit.gwi.uni-muenchen.de/?p=12756\&v3> [ultimo accesso il 29/09/2019].

RAIMONDI Gianmario, 2019b, «Atlanti interpretativi, cartografia sintetica, distanza linguistica. Il banco di prova dell'APV-Atlas des patois valdôtains», Géolinguistique, $\mathrm{n}^{\circ} 19$. Pubblicato online il 9 dicembre $2019<\mathrm{http}$ //journals.openedition.org/geolinguistique/1170> [ultimo accesso il 10/10/2021].

REGIS Riccardo, 2019, «Sul tipo lessicale», Géolinguistique, $\mathrm{n}^{\circ}$ 19. Pubblicato online il 9 dicembre $2019 \mathrm{su}<\mathrm{http}$ ://journals.openedition.org/geolinguistique/1229> [ultimo accesso il 10/10/2021].

TUAILLON Gaston, 1972-1973, Questionnaire pour enquêtes dialectales en pays alpin, Grenoble, ELLUG.

\section{NOTE}

1. Una copia pdf del volume è scaricabile gratuitamente al seguente indirizzo web: $<$ www.patoisvda.org/atlas-patois-valdotains/>. Tutto il materiale d'archivio APV (registrazioni e quaderni d'inchiesta) è di proprietà dell'Assessorato Regionale ed è conservato al BREL (Aosta, via Croce di Città 16), cui si può anche richiedere la versione a stampa del volume (brel@regione.vda.it).

2. Atlas des patois valdôtains: regards croisées / sguardi incrociati, convegno online del 6 maggio 2021 $<$ www.univda.it/eventi-univda/latlas-des-patois-valdotains-regards-croises-sguardi-incrociatipresentazione-del-primo-volume/>. Al convegno (che ha registrato 120 partecipanti da Italia, Francia, Svizzera e Germania) sono intervenuti Roland Bauer (Salisburgo), Yan Greub (Neuchâtel), Thomas Krefeld (Monaco di Baviera), Lorenzo Massobrio (Torino), Elton Prifti (Vienna), Matteo Rivoira (Torino), Giovanni Ruffino (Palermo), Glauco Sanga (Venezia), Tullio Telmon (Torino), Alessandro Vitale Brovarone (Torino). Gli atti (a cura di G. Raimondi e P. B. Mas) sono in corso di pubblicazione.

3. Si rimanda qui anche alla rassegna bibliografica dei contributi scientifici preparatori prodotti dall'APV nel periodo 1972-2017 reperibile in Raimondi (2019a).

4. Nell'articolazione geolinguistica dell'area valdostana si oppone tradizionalmente una sub-area occidentale (detta comunemente «Alta Valle») e una orientale («Bassa Valle»), i cui confini sono 
determinati da una serie di isoglosse nord-sud che attraversano con andamento variabile la Conca di Châtillon, dove la Dora cambia l'orientamento del suo corso da ovest-est a nord-sud (Raimondi, 2019). A questa ripartizione generale si deve necessariamente aggiungere la considerazione delle dinamiche di conservazione e innovazione fra valle centrale e valli laterali, tipiche di tutte le aree montane e ormai sufficientemente formalizzate dalle più recenti teorie generali sulla Mountain Linguistics (cfr. ad es. Nichols, 2015). Su alcune altre sub-aree fonetiche si tenga poi presente Keller (1958).

5. L'impostazione offre addentellati con la caratterizzazione storica dell'area. La castellania di Quart (che comprendeva tutto il retroterra montano settentrionale di Aosta fino alla Valpelline, incluse le località sul versante sinistro della valle del torrente Buthier, che conduce al Gran San Bernardo; cfr. Raimondi, 2012) era infatti la più grande fra quelle che, dalla fine del XIII secolo in avanti, erano sotto il controllo diretto dei Savoia. Aosta (che era governata piuttosto autonomamente, in un regime di franchigie concesse dai Savoia ai cittadini e al Vescovo di Aosta) faceva invece parte della Castellania di Valdigne (Alta Valle).

6. Fra le isoglosse fonetiche si segnalano qui gli esiti dei nessi CONS. BILABIALE+/l/ (tipi /tabla/ vs. /tabja/, su cui cfr. anche Keller, 1958: 123 per l'estensione del tipo italoromanzo nell'area mediana sul versante sinistro della Dora) e /s/+/t/ (/teta/ vs. /tehta/) e il trattamento di CAiniziale (/tsa/ vs. / $\mathrm{t} \mathrm{Sa}$ /; Raimondi, 2014: 783), il cui andamento riportiamo in fig. 5. Per altre isoglosse, di carattere lessicale, si rimanda ancora a Raimondi (2014: 782-785 e fig. 2).

7. Nel dibattito teorico sulla tipizzazione riassunto ed esemplificato in Regis (2019), la soluzione adottata dall'APV rientra dunque nel caso 3. della prassi ALEPO («tipo dialettale di sintesi»), che a sua volta è conforme alla 2. («forme dialettali di riferimento [...] registrate nei dizionari») dell'Index zum AIS. Il tipo ideale si basa, quando possibile, sulla forma presente nel più ampio dizionario di riferimento per il patois centrale (Chenal \& Vautherin, 1997). L'adozione esclusiva di questo modello, nel caso dell'APV, è naturalmente reso possibile dall'appartenenza delle parlate rappresentate a un unico gruppo linguistico.

8. Queste le voci relative contenute nel Petit glossaire des termes de linguistique dell'atlante: «MotIVATION (SÉMANTIQUE): relation conceptuelle qui explique l'adoption d'un *LEXèME comme *ICÔNYME pour la formation d'un mot. La ressemblance de forme est par ex. la motivation pour l'adoption de \{lune\} comme icônyme pour le fr. lunettes» (APV/1, 2020: 16); «ICÔNYME : *LEXÈME motivé sémantiquement qui est utilisé pour la création d'un nouveau mot. En français, le lexème \{lune\}, motivé par la ressemblance de la forme, est par ex. l'icônyme de lunettes» (APV/1, 2020: 17).

9. La richiesta del nome del filtro in materiale vegetale dell'antico imbuto da latte ha orientato di volta in volta gli informatori sul nome dell'oggetto (il 'fascio' di erba utilizzato a questo fine, denominato frota, frotòn o tortsòn; iconimo \{faisceau\}) o sul materiale di cui esso era fatto (dal generico rèis 'radici' o pé 'radici fini', lett. 'peli', iconimo \{racines\}; al nome della pianta gramòn 'gramigna', iconimo \{chiendent\}), attraverso un procedimento metonimico.

10. In questo caso, risposte traduttive (vèyé, chorveyè, per $\{($ sur)veiller\}), variamente sinonimiche (*aveitsé < germ. *WAHTA, *bucà < lat. BATARE; 3. mirà < lat. *MIRARE, corrispondenti diatopicamente diversificati per \{regarder\}, o aguètté, sempre < germ. ${ }^{*}$ wahta, per \{observer\}) o orientate (in cinque casi) sulla perifrasi \{faire attention\}, compongono un quadro molto variato di risposta allo stimolo originale. 


\section{RIASSUNTI}

In occasione della pubblicazione del primo volume dell'APV-Atlas des patois valdôtains (2020), il contributo illustra gli elementi salienti della sua struttura editoriale e scientifica, soffermandosi in particolare sulla copertura geolinguistica dell'APV in rapporto agli atlanti nazionali preesistenti, sulle metodologie di elaborazione della sua griglia referenziale particolarmente dettagliata e sugli apparati degli indici, e concludendo con un approfondimento sull'approccio semantico "motivazionale", utilizzato, insieme a quelli più tradizionali, nella cartografia sintetica.

À l'occasion de la publication du premier volume de l'APV - Atlas des patois valdôtains (2020), l'article illustre les éléments saillants de sa structure éditoriale et scientifique, en abordant en particulier la couverture géolinguistique de l'APV par rapport aux atlas nationaux préexistants, les modalités d'élaboration de sa grille référentielle très détaillée et les apparats des indexes. Nous concluons par un approfondissement sur l'approche sémantique "motivationnelle», utilisée, avec ceux plus traditionnels, dans la cartographie de synthèse.

Following the publication of the first volume of the APV - Atlas des patois valdôtains (2020), this paper outlines the main elements of its editorial and scientific structure, dwelling particularly on its geolinguistic coverage in comparison to previous national atlases, on the elaboration methodologies of its highly detailed referential grid and of its indexes, concluding then with an insight on the "motivational" semantic approach that, together with the more traditional ones, has been used in the synthetic cartography.

\section{INDICE}

Mots-clés : atlas linguistiques, Val d'Aoste, francoprovençal, motivation sémanthique

Keywords : linguistic atlases, Aosta Valley, Franco-Provençal, semantic motivation

Parole chiave : atlanti linguistici, Valle d'Aosta, francoprovenzale, motivazione semantica

\section{AUTORE}

GIANMARIO RAIMONDI

Università della Valle d'Aosta

g.raimondi@univda.it 